\title{
An evolutionarily conserved transcriptional response to viral infection in Caenorhabditis nematodes
}

\author{
Kevin Chen, Carl J. Franz, Hongbing Jiang, Yanfang Jiang and David Wang*
}

\begin{abstract}
Background: Caenorhabditis elegans is a powerful model organism for probing many biological processes including host-pathogen interactions with bacteria and fungi. The recent identification of nematode viruses that naturally infect C. elegans and Caenorhabditis briggsae provides a unique opportunity to define host-virus interactions in these model hosts.

Results: We analyzed the transcriptional response of pathogen infected C. elegans and C. briggsae by RNA-seq. We identified a total of 320 differentially expressed genes (DEGs) in C. elegans following Orsay virus infection. The DEGs of known function were enriched for ubiquitin ligase related genes; however, the majority of the genes were of unknown function. Interestingly, many DEGs that responded to Orsay virus infection were similar to those induced by Nematocida parisii infection, which is a natural microsporidia pathogen of C. elegans that like Orsay virus infects intestinal cells. Furthermore, comparison of the Orsay virus DEGs in C. elegans to Santeuil virus DEGs in C. briggsae identified 58 C. elegans genes whose orthologs were likewise differentially expressed in C. briggsae, thereby defining an evolutionarily conserved response to viral infection.
\end{abstract}

Conclusions: The two different species C. elegans and C. briggsae, which diverged $\sim 18$ million years ago, share a common set of transcriptionally responsive genes to viral infection. Furthermore, a subset of these genes were also differentially expressed following infection by a eukaryotic pathogen, N. parisii, suggesting that these genes may constitute a broader pan-microbial response to infection.

Keywords: Host-virus interaction, Orsay virus, Santeuil virus, N. parisii, C. elegans, C. briggsae, Pathogen infection, Transcriptional profile

\section{Background}

Caenorhabditis elegans is a model organism widely used to interrogate host-pathogen interactions $[1,2]$. In recent years, studies in $C$. elegans have identified genes that are essential for immunity against bacterial and fungal pathogens. For instance, roles for p38 MAP kinase [3], TGF- $\beta$ [4], DAF-2/DAF-16 insulin-like receptor signaling [5], and the transcription factor zip-2 [6] have been established in protection against bacterial or fungal infections in $C$. elegans. In addition, multiple studies have dissected the $C$. elegans transcriptional response to a range of different pathogens including Bacillus thuringiensis

\footnotetext{
* Correspondence: davewang@wustl.edu

Departments of Molecular Microbiology and Pathology and Immunology, Washington University in St. Louis, School of Medicine, 660 S. Euclid Avenue, St, Louis, MO, USA
}

[7], Pseudomonas aeruginosa and Staphylococcus aureas [8], Serratia marcescens, Enterococcus faecalis, Erwinia carotovora, and Photorhabdus luminescens [9], and fungal pathogens including Drechmeria coniospora [10], Harposporium sp. [11] and Nematocida parisii [12]. There is some overlap in the transcriptional responses to the various bacterial and fungal infections, suggesting that $C$. elegans maintains both "pan-microbial" and "microbe-specific" repertoires of pathogen response genes [13]. From the transcriptionally induced genes, some functional immune response genes have been identified and characterized.

Much less is understood about host responses in $C$. elegans to viral infection, largely due to the lack of, until recently, a natural virus capable of infecting C. elegans. Previous studies using artificial viral infection conditions 
with vaccinia virus [14], nematode cells with vesicular stomatitis virus $[15,16]$ or a transgenic virus replicon system (Flock house virus) [17] have demonstrated antiviral roles for the programmed cell death genes ced-3 and ced-4, and RNA interference (RNAi) pathways in C. elegans. With the discovery of Orsay virus, the first known natural viral pathogen of C. elegans, RNAi and ubiquitin-mediated protection against viral infection have been described [12, 18-23].

In addition to Orsay virus, two related viruses, Santeuil and Le Blanc, were discovered in wild Caenorhabditis briggsae strains. Orsay virus only infects C. elegans while Santeuil virus and Le Blanc virus only infect $C$. briggsae $[18,24]$. All three viruses have a common tissue tropism and specifically infect the intestine [25]. The identification of multiple viruses that infect two host species that diverged $\sim 18$ million years ago affords the unique opportunity to define evolutionarily conserved host responses to viral infection [26]. Furthermore, C. elegans can also be infected specifically in the intestine by the microsporidia $N$. parisii [27]. Thus, host responses to these various microbial pathogens can be compared and contrasted. In this study, to define the transcriptional response to these natural pathogens, we used highthroughput RNA sequencing (RNA-seq) to quantify the host mRNA levels following different microbial infections. Collectively, these results shed light on the host response to viral infection and provide insight into the larger context of antimicrobial defense in C. elegans.

\section{Results}

C. elegans transcriptional response to Orsay virus infection To define the transcriptional changes in C. elegans upon Orsay virus infection, we compared RNA-seq results from infected and non-infected animals. We analyzed both the laboratory reference strain $\mathrm{N} 2$ as well as the $r d e-1$ mutant, which bears a mutation in the Argonaut protein RDE-1 that is part of the RNAi pathway. The rde-1 mutant sustains higher levels of Orsay virus replication and accumulate 100 -fold more viral RNA compared to N2 [18] enabling us to assess the impact of more robust viral infection levels, as well as a defective RNAi pathway, on the transcriptional response. Samples were analyzed at $12 \mathrm{~h}$ post infection (hpi), a time by which Orsay virus protein expression is observed in most $r d e-1$ animals [25]. We used the edgeR package [28] to identify differentially expressed genes (DEGs) in both $\mathrm{N} 2$ and $r d e-1$ strains $(\mathrm{n}=$ 3 replicates for each, FDR $<0.05$, Table 1 ). The vast majority of the DEGs were up-regulated, while a small subset of DEGs were down-regulated (Table 1, Additional file 1). Among the induced genes, up-regulation ranged between 1.8-fold to over 1000-fold compared to mock control (Additional files 1 and 2).
Table 1 The number of C. elegans differentially expressed genes (DEGs) upon different pathogen infection

\begin{tabular}{llll}
\hline & $\begin{array}{l}\text { Orsay virus } \\
{[\mathrm{N} 2]}\end{array}$ & $\begin{array}{l}\text { Orsay virus } \\
{[\text { rde-1] }}\end{array}$ & $\begin{array}{c}\text { N. parisii } \\
{[\mathrm{N} 2]}\end{array}$ \\
\hline DEGs UP & 129 & 277 & 185 \\
$\begin{array}{l}\text { DEGs DOWN } \\
\begin{array}{l}\text { DEGs Shared with Orsay } \\
\text { virus [N2] }\end{array}\end{array}$ & 1 & 21 & 11 \\
$\begin{array}{l}\text { DEGs shared with Orsay } \\
\text { virus [rde-1] }\end{array}$ & 108 & 108 & 108 \\
\hline
\end{tabular}

Differentially expressed genes were analyzed using edge $R$ with 3 replicates and a FDR $<0.05$ cutoff. N/A: Not applicable

Between the two different strains of $C$. elegans, there were 108 DEGs shared, while there were 22 and 190 DEGs specific to $\mathrm{N} 2$ and $r d e-1$, respectively (Fig. 1). The majority of the DEGs were of unknown function. For the subset that had annotations, we identified several enriched gene families and functions using the software package DAVID [29, 30], (FDR $<0.05$, Table 2). Both $\mathrm{N} 2$ and $r d e-1$ DEGs were enriched for several gene families including DUF38 domain genes, DUF713 domain genes, MATH (meprin-associated Traf homology) domain genes and a family of paralogs exemplified by $\mathrm{C} 17 \mathrm{H} 1.3$ ( $\mathrm{C} 17 \mathrm{H} 1$ family genes hereafter, named after the $\mathrm{C} 17 \mathrm{H} 1$ locus which contains the largest number of genes in this family) (Table 2). The C17H1 family genes have an ortholog in human, Amyotrophic Lateral Sclerosis 2 Chromosome Region Candidate 12 (ALS2CR12) which encodes a protein of unknown function. Most of the DUF38 domain containing genes also contain an F-BOX domain, which is associated with the ubiquitin ligase pathway. The DUF713 domain genes are specific to the Caenorhabditis genus and do not have any associated functions. Of the 22 DEGs that were specific to N2 infected with Orsay virus, no statistically enriched gene families were identified. Of the 190 rde-1 specific DEGs, there were several additional enriched gene families including CUB-like domain genes, CUB domain genes, and zinc finger (C6HC-type) domain genes. In addition, innate immune response genes were enriched based on GO annotation (Table 2). For the DEGs that were shared between the two strains, $r d e-1$ DEGs in general were induced to a greater degree (Additional files 1 and 2).

To confirm the RNA-seq results, we used quantitative real-time reverse transcription PCR (qRT-PCR) of an independent Orsay virus infection in the N2 strain to evaluate transcript levels of three highly up-regulated genes (C17H1.3, C17H1.8, and F26F2.1) and two genes that did not change following viral infection (B0024.4 and tsp-1). All five genes yielded similar results between transcriptional profiling and qRT-PCR (Additional file 3). 


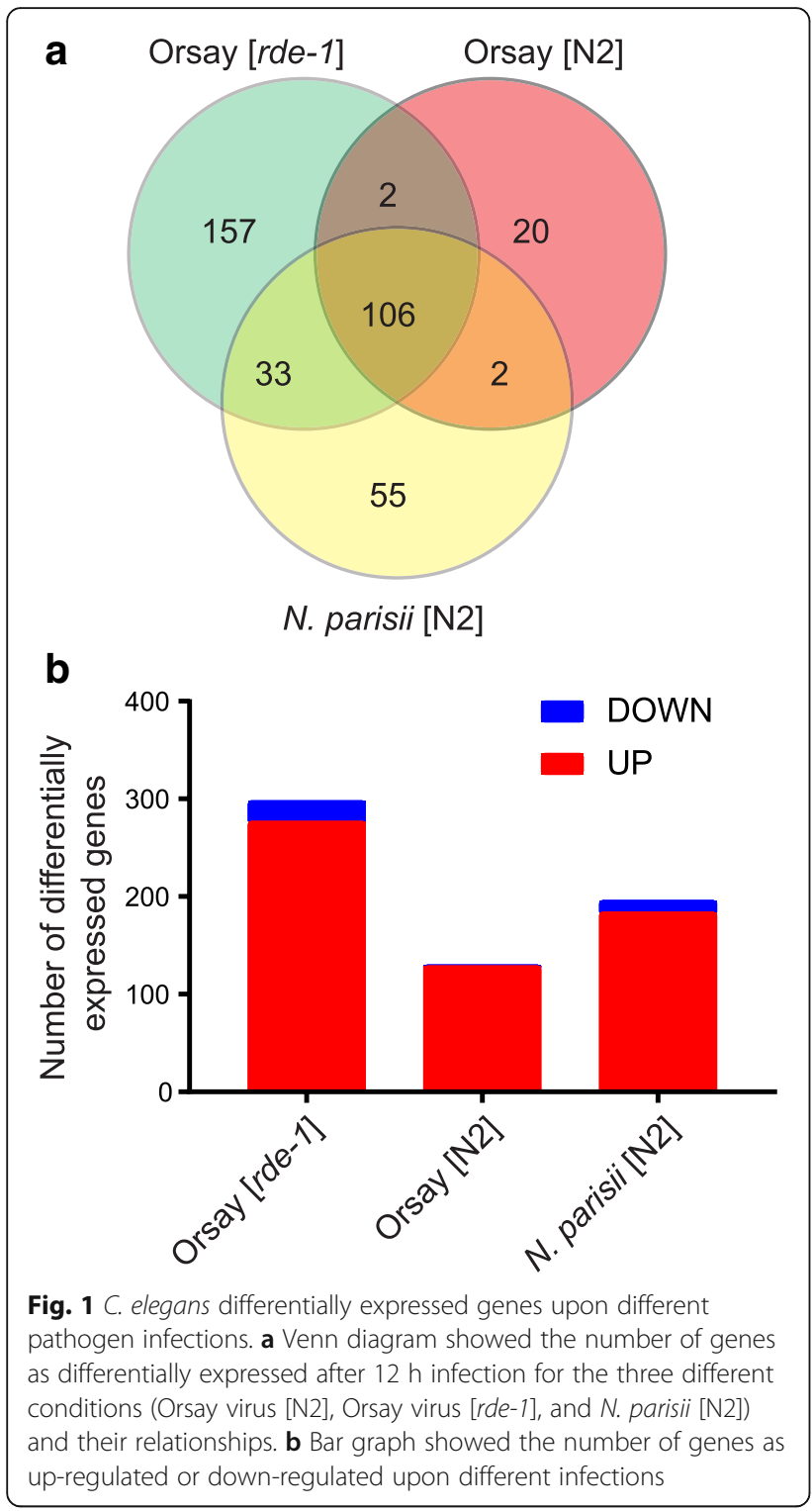

\section{Orsay virus and $N$. parisii induced a shared transcriptional response}

Because the microsporidia $N$. parisii is also an intracellular pathogen of intestinal cells in C. elegans, we performed a parallel transcriptional profiling of $N$. parisii infection in N2. There were 196 DEGs identified in N2 at 12 hpi of $N$. parisii (edgeR, $\mathrm{n}=3$ replicates, FDR < 0.05 , Table 1 ); notably 108 DEGs were shared with Orsay virus infection of N2 (Fig. 1, Table 1). Thus, the majority of the Orsay virus induced DEGs in N2 were also differentially expressed following $N$. parisii infection. Another 33 DEGs were shared between $N$. parisii infection of N2 and Orsay infection of the $r d e-1$ strain (Fig. 1). Interestingly, only two genes were down-regulated in both $r d e-1$ upon Orsay virus infection and $\mathrm{N} 2$ upon $N$. parisii infection. The two genes, pud-1.2, and pud-4, are paralogs known to be regulated by DAF-2, an insulinlike receptor $[31,32]$. Of the 55 DEGs specific to N2 infected with $N$. parisii, the enriched gene families included zinc finger (RING-type) domain genes and Ctype lectin-like genes (Table 2). We compared our results with a recently published expression profile of $N$. parisii infection, which was performed in a different genetic background [12], and found the majority of the up-regulated genes that we identified were also upregulated at $8 \mathrm{~h}$ post infection in the previous publication (Additional files 4 and 5).

There were four gene families enriched across all infection conditions in C. elegans: Orsay virus [N2], Orsay virus [rde-1], and N. parisii [N2] (Table 2). F-box domain genes (DUF38) and MATH domain genes are adapter proteins, which encode a Cullin-binding domain and a substratebinding domain that target proteins for E3 ubiquitinligase mediated proteolysis [33]. There were a total of 35 unique ubiquitin ligase adaptor genes that were highly upregulated (between 4-fold to 1000 -fold), 8 of which were induced in all three conditions. Specifically, F-box proteins act in concert with other proteins that are members of the Skp/Cullin/F-box (SCF) complex to facilitate ubiquitinligase mediated proteolysis. Interestingly, $s k r-4$, a SCF complex gene was up-regulated in all three infection conditions. Furthermore, in the rde-1 Orsay virus infected condition, two additional SCF complex genes $s k r-5$ and cul-6 were up-regulated.

The C17H1 family genes and DUF713 domain genes have no known functions. For both the C17H1 family genes and DUF713 domain genes, more than $50 \%$ of the family members were differentially expressed. The C17H1 gene family has a total of 36 members in $C$. elegans, and of those the same 25 members (except for F22G12.7 in Orsay virus [N2] condition that was not statistically significant) were up-regulated following both Orsay virus and $N$. parisii infection (Fig. 2, Table 2). The DUF713 domain genes have a total of 10 members in $C$. elegans and have from 5-9 members of the gene family up-regulated following pathogen infection (Fig. 2, Table 2). The C17H1 family had the most DEGs represented in the Orsay virus and $N$. parisii infections (Table 2), and some of the genes were among the highest induced with close to 1000-fold increase compared to mock infection. Given the highly distinct nature of Orsay virus from the eukaryotic microsporidium $N$. parisii, this shared transcriptional response may represent a cellular stress pathway in $C$. elegans triggered by intracellular perturbation.

\section{Evolutionarily conserved response to viral infection in C. elegans and C. briggsae}

We next defined the DEGs in C. briggsae following Santeuil virus infection. Because the $C$. briggsae laboratory 
Table 2 Gene Ontology (GO), InterPRO term enriched from C. elegans genes differentially expressed upon Orsay virus or N. parisii infection

\begin{tabular}{|c|c|c|c|c|c|}
\hline Condition & Go term, InterPRO classification & Human ortholog & Functional annotations & Gene count & FDR \\
\hline \multirow[t]{4}{*}{ Orsay virus [N2] } & IPR026674:ALS2CR12 protein (C17H1 family) & Yes & N/A & 24 & $6.9 \mathrm{E}-32$ \\
\hline & IPR002900:DUF38 & No & F-box associated & 13 & 6.6E-09 \\
\hline & IPR007883:DUF713 & No & N/A & 5 & $1.4 \mathrm{E}-06$ \\
\hline & IPR002083:MATH & Yes & MATH & 8 & $1.3 \mathrm{E}-06$ \\
\hline \multirow[t]{8}{*}{ Orsay virus [rde-1] } & IPR026674:ALS2CR12 protein (C17H1 family) & Yes & N/A & 25 & 2.3E-25 \\
\hline & GO:0045087:Innate immune response & N/A & Innate immunity & 23 & $6.2 \mathrm{E}-13$ \\
\hline & IPR002083:MATH & Yes & MATH & 14 & 3.1E-09 \\
\hline & IPR007883:DUF713 & No & N/A & 7 & 2.9E-08 \\
\hline & IPR002900:DUF38 & No & F-box associated & 16 & $3.8 \mathrm{E}-06$ \\
\hline & IPR003366:CUB-like domain & No & N/A & 9 & 4.7E-06 \\
\hline & IPR000859:CUB domain & Yes & CUB & 8 & $6.1 \mathrm{E}-04$ \\
\hline & IPR002867:Zinc finger, C6HC-type & Yes & Ubiquitin related & 4 & 2.1E-02 \\
\hline \multirow[t]{8}{*}{ N. parisii [N2] } & IPR026674:ALS2CR12 protein (C17H1 family) & Yes & N/A & 25 & $1.4 \mathrm{E}-29$ \\
\hline & IPR007883:DUF713 & No & N/A & 9 & 1.7E-14 \\
\hline & IPR002083:MATH & Yes & MATH & 11 & $3.0 \mathrm{E}-08$ \\
\hline & IPR002900:DUF38 & No & F-box associated & 13 & 2.5E-06 \\
\hline & GO:0045087:Innate immune response & N/A & Innate immunity & 8 & $5.2 \mathrm{E}-03$ \\
\hline & IPR001841:Zinc finger, RING-type & Yes & Zinc finger & 8 & $5.9 \mathrm{E}-03$ \\
\hline & IPR016186:C-type lectin-like & Yes & C-type lectin-like & 9 & $1.0 \mathrm{E}-02$ \\
\hline & GO:0005764:lysosome & N/A & lysosome & 4 & 2.9E-02 \\
\hline
\end{tabular}

GO term, InterPRO classification enrichment was analyzed using online DAVID Bioinformatics Resources 6.8. Bold denotes conserved terms across the three infection conditions: Orsay virus [N2], Orsay virus [rde-1], and N. parisii [N2]. N/A: Not applicable

reference strain AF16 does not support Santeuil virus replication in our hands, we used the wild C. briggsae isolate JU1264 which we had previously demonstrated to be susceptible to Santeuil virus infection [18]. In $C$. briggsae, there were 258 DEGs following infection by Santeuil virus (edgeR, $\mathrm{n}=3, \mathrm{FDR}<0.05$, Additional file 2). Of the Santeuil virus DEGs, 37 were downregulated and 221 were up-regulated. On a technical note, JU1264 sequence reads were mapped to the closely related AF16 reference transcriptome; strain specific sequence differences may lead to incomplete mapping to some genes and thus a potential underestimate of the DEGs.

To confirm the RNA-seq results, we used qRT-PCR of an independent Santeuil virus infection of JU1264 to evaluate transcript levels of two up-regulated genes: CBG03198, a gene with C. elegans orthologs that were also up-regulated, and $C B G 06596$, an ortholog of the C17H1 family in C. briggsae. The two genes yielded similar results between transcriptional profiling and qRT-PCR (Additional file 6).

Approximately $60 \%$ of all genes in C. briggsae have well-defined orthologs in C. elegans [34]. We further compared the DEGs in C. elegans to their orthologous genes in C. briggsae. Of the 320 genes identified as differentially expressed in either $\mathrm{N} 2$ or $r d e-1$ or both (union of $\mathrm{N} 2$ and $r d e-1$ viral infection induced DEGs), 197 have orthologs in C. briggsae. 59 of these had C. briggsae orthologs that were also differentially expressed following Santeuil infection (Fig. 3, Additional file 7). The majority (57 of 59) of the DEGs were up-regulated in both species. One gene, hmit-1.1, was repressed in both the Orsay virus [rde-1] and the Santeuil virus [JU1264] conditions while clec-7 was repressed in Orsay virus [rde-1] and induced in Santeuil virus [JU1264]. In total, there were 58 DEGs in the conserved response to viral infection. 29 C. elegans genes were induced in both N2 and rde-1 and had corresponding C. briggsae orthologs induced following Santeuil infection. These, included 14 C17H1 family genes, four DUF713 domain genes, a gene in the RNAi pathway: C04F12.1, and a gene downstream of daf-16: dod23. The remaining 9 genes have no known functions. There were two C. elegans DEGs in N2 (but not in $r$ de-1) whose orthologs in C. briggsae were also DEGs. These were F14F9.3, a zinc finger (C6HC-type) domain gene and ZK177.8, the human ortholog of which is SAMHD1, an antiviral gene against human immunodeficiency virus 1 [35]. Finally, there were 27 C. elegans DEGs in $r d e-1$ (but not N2) with corresponding C. briggsae DEGs. Six were immune related genes, four were zinc finger (C6HC-type) domain 


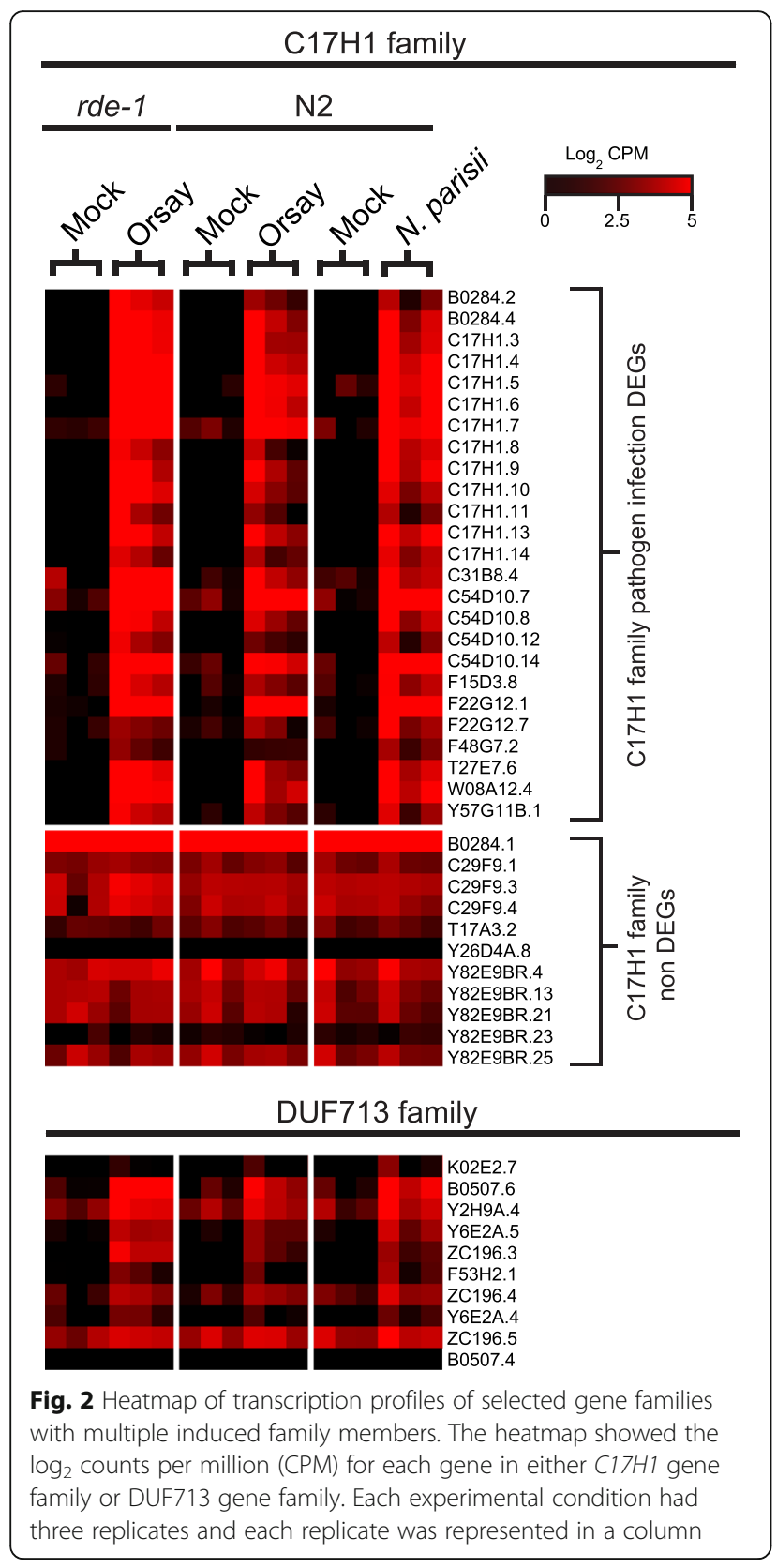

genes, three were transcription factors (zip-1, zip-5 and zip10 ), one was an RNAi related gene (sid-5) and the remainder had varying annotations (Additional file 7).

Interestingly, the orthologs in C. briggsae of the DUF38 genes and MATH domain genes that responded to both Orsay virus and $N$. parisii infection were not differentially expressed following Santeuil virus infection. This suggests that the induction of F-box and MATH genes may be a $C$. elegans specific transcriptional response. (Additional file 7).

\section{Evolutionarily conserved pan-microbial response}

In total, 37 of the 58 conserved viral DEGs were also differentially regulated following $N$. parisii infection (Fig. 3).

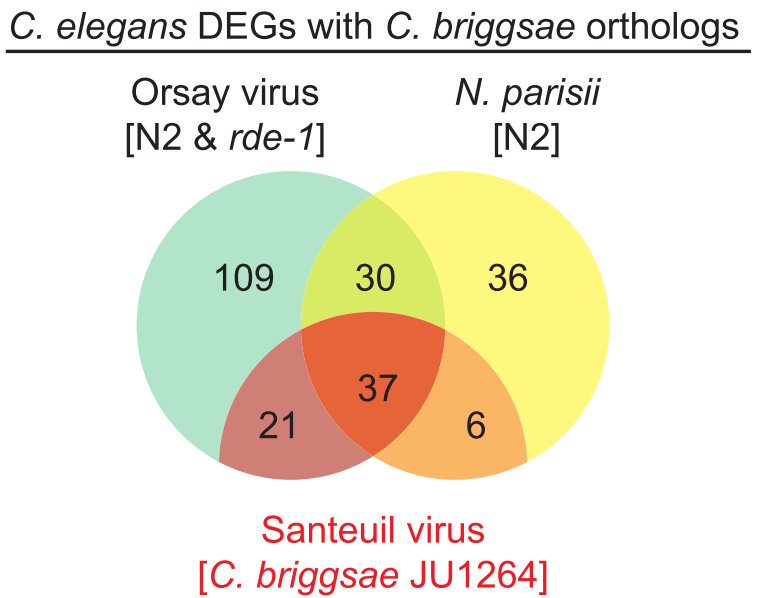

Fig. 3 Evolutionarily conserved pan-microbial responsive C. elegans genes. C. elegans genes differentially expressed following Orsay and $N$. parisii infection that have orthologs in C. briggsae were differentially expressed. Red shading indicates C. elegans genes whose orthologs in C. briggsae have conserved response to Santeuil infection of C. briggsae

Strikingly, all of the 29 DEGs that were conserved between the three viral infection conditions, Orsay virus [N2], Orsay virus [rde-1], and Santeuil virus [JU1264], were also $N$. parisii induced DEGs. Specifically, members of the $\mathrm{C} 17 \mathrm{H} 1$ and DUF713 gene families appeared to be pan-microbial response genes while the zinc finger (C6HC-type) gene responses were specific to viral infection (Additional file 7). To further assess the potential roles of the conserved virus induced DEGs in the context of different pathogens, we compared our current results with previous published studies of pathogen induced host response. A prior microarray study of Orsay virus infection of $C$. elegans identified multiple DEGs [19], of which 24 DEGs were shared between the two studies in N2 background and 40 DEGs were shared in the rde-1 mutant (Additional files 4 and 5). In addition, we identified additional statistically significant viral DEGs, possibly due to the use of synchronized animals. We examined a panel of representative bacterial pathogens including Pseudomonas aeruginosa, Enterococcus faecalis, Staphylococcus aureus, Bacillus thuringiensis, Serratia marcescens, and Photorhabdus luminescens [7, 8, 11, 36] and fungal pathogens including Drechmeria coniospora and Harposporium sp. [11]. All up-regulated and downregulated DEGs identified from previous studies were compared to the up-regulated or down-regulated Orsay virus DEGs. P. aeruginosa, P. luminescens and D. coniospora infection each shared a significant number of DEGs with Orsay virus infection (Fisher exact test $p<0.001)$. Notably, dod-22 was differentially expressed in all four instances. By contrast, there was an inverse association of E. faecalis DEGs with Orsay 
virus DEGs (Fisher exact test $p<0.001$ ), with fewer shared DEGs than expected by chance. Finally, the other pathogens did not have a significant relationship with the Orsay virus DEGs (Table 3).

We also specifically investigated the transcriptional response of the $\mathrm{C} 17 \mathrm{H} 1$ gene family members following infection by other pathogens. In prior published studies, $P$. luminescens and D. coniospora each induced multiple of the virally up-regulated $\mathrm{C} 17 \mathrm{H} 1$ gene family members (Table 3). Interestingly, some of the $\mathrm{C} 17 \mathrm{H1}$ family genes that were not up-regulated following viral infection were differentially expressed following infection by $D$. coniospora, Harposporium, P. luminescens, and S. marcescens (Table 3). Thus, there may be pan-microbial responsive $\mathrm{C} 17 \mathrm{H} 1$ family members as well as those that respond to specific pathogens.

\section{Discussion}

We defined the host transcriptional response to viral infection in C. elegans and C. briggsae. From our statistical analysis of Orsay virus infections in $\mathrm{N} 2$ and $r d e-1 \mathrm{mu}-$ tant strains, we identified a total of 320 DEGs in C. elegans, of which 108 DEGs were shared. In the rde-1 Orsay virus infection, there were more DEGs compared to infection of N2. In addition, the magnitude of the transcriptional changes in $r d e-1$ was generally greater. One possible explanation for this observation is that the higher levels of viral infection in $r d e-1$ may have created a more significant perturbation from the basal state, leading to a more robust transcriptional response. Alternatively, the lack of competent RNAi in $r d e-1$ may have resulted in induction of a distinct, compensatory host response. One potential limitation of these studies is that Orsay, Santeuil, and N. parisii infection is thought to be limited to at most the 20 intestinal cells present in Caenorhabditis nematodes. Because our transcriptional profiling used RNA extracted from populations of entire animals (each C. elegans has 959 somatic cells), some transcriptional responses may have been masked by the basal level of transcription in the uninfected cells, and thus our results are likely an underestimate of the transcriptional changes occurring in the intestinal cells.

Strikingly, 108 of the N2 DEGs were also differentially expressed following infection with the microsporidium, N. parisii (Fig. 1). Orsay virus is a small single stranded RNA virus with a bipartite genome of $3.6 \mathrm{~Kb}$ and $2.6 \mathrm{~Kb}$ that is only known to encode three proteins [18]. By contrast, $N$. parisii has a $4.1 \mathrm{Mb}$ genome and encodes more than 2000 genes [27, 37]. Despite the lack of obvious similarity between these two microbes, the fact that a significant fraction of the transcriptional response to these two pathogens overlapped suggests that C. elegans may have some form of a universal "stress response". One clear commonality between the two is that they are both intracellular intestinal pathogens of C. elegans; in fact they are the only intracellular pathogens of $C$. elegans described to date. Thus, the conserved transcriptional response may reflect recognition of some shared intracellular perturbation. Interestingly, although some of these shared response genes are potentially involved in the ubiquitin ligase pathway, the majority of the shared response genes are largely unannotated genes of unknown function. These genes could play important roles in immunity against pathogen infection. Alternatively, it is also possible that these genes are important for pathogen infection, and that the pathogen alters the transcriptional response to facilitate infection and replication.

Many of the characterized genes induced by Orsay virus or $N$. parisii infection in C. elegans were genes in the ubiquitin ligase pathway. When challenged with either Orsay virus or $N$. parisii, there were 35 unique Fbox related or MATH domain genes up-regulated. In addition, SCF complex genes, such as $s k r-4$, were upregulated in all $C$. elegans infections while $s k r-5$ and cul6 were up-regulated in the $r d e-1$ mutant infected with Orsay virus. Most of the F-box and MATH family members have sites in their substrate binding domains

Table 3 Conserved responses between bacterial or fungal pathogen to Orsay virus DEGs

\begin{tabular}{llllll}
\hline & Total DEGs & $\begin{array}{l}\text { Bacterial/fungal DEGs } \\
\text { shared with Orsay DEGs }\end{array}$ & Overlap significance & $\begin{array}{l}\text { Number of DEGs from } \\
\text { C17H1 family }\end{array}$ & $\begin{array}{l}\text { Number of C17H1 DEGs shared } \\
\text { with Orsay virus infection }\end{array}$ \\
\hline B. thuringiensis & 246 & 5 & $4.4 \mathrm{E}-01$ & 0 & 0 \\
D. coniospora & 3787 & 84 & $6.1 \mathrm{E}-04$ & 15 & 11 \\
E. faecalis & 3819 & 40 & $3.7 \mathrm{E}-03$ & 2 & 2 \\
Harposporium sp. & 3695 & 60 & $7.1 \mathrm{E}-01$ & 6 & 4 \\
P. aeruginosa & 146 & 10 & $1.0 \mathrm{E}-04$ & 0 & 0 \\
P. luminescens & 3797 & 111 & $6.4 \mathrm{E}-12$ & 12 & 11 \\
S. aureus & 386 & 9 & $2.1 \mathrm{E}-01$ & 1 & 1 \\
S. marcescens & 3384 & 56 & $6.5 \mathrm{E}-01$ & 11 & 7 \\
\hline
\end{tabular}

Pathogen infection expression profile analysis data were obtained from $[7,8,11,36]$. The DEGs from each pathogen were compared to Orsay virus DEGs. Association significance is calculated using Fisher exact test. Bold indicates a significant negative correlation 
that are under strong positive selection and are greatly expanded in C. elegans in comparison to humans [33]. This suggests a possible role of ubiquitin ligase as part of the C. elegans host-pathogen interaction to restrict pathogen proliferation. Indeed, SCF ubiquitin ligases are demonstrated as a line of defense against infection by Orsay virus and N. parisii in C. elegans [12]. Intriguingly, none of the DEGs in C. briggsae were known F-box or MATH genes, suggesting that these ubiquitin ligase pathways may be a specific $C$. elegans response.

There are varying degrees of conservation between Orsay virus response genes to other pathogens of $C$. elegans. We analyzed previously published transcriptional profiling studies of infection by 8 bacterial and fungal pathogens and identified three that have a significant fraction of DEGs shared with Orsay virus infection. The three pathogens, $P$. aeruginosa, $P$. luminescens and $D$. coniospora, all can affect the intestine of the worm, but each does so in unique fashion. $P$. aeruginosa PA14 primarily kills by excreted toxins, $P$. luminescens colonizes the intestinal lumen, which is characterized by the appearance of cytosolic crystalline structures of unknown origin [38], and D. coniospora produces threadlike hyphae that penetrate and eventually kill the infected animal [39]. Other pathogens that also target the intestine such as E. faecalis and S. aureus did not have significant DEGs in common with Orsay virus infection, demonstrating a specificity of the host response. The different responses of $C$. elegans to various pathogens suggest the existence of distinct sensing and regulatory mechanisms. One potential regulatory element in response to virus infection is $d r h-1$, a RIG-I like protein in $C$. elegans. Previous studies have determined that $d r h-1$ both acts directly as a effector in the RNAi pathway to restrict virus replication and as a sensor of virus infection critical for downstream host responses $[19,20]$.

Comparative analysis of the DEGs in virus infected $C$. elegans and C. briggsae identified 58 C. elegans genes whose $C$. briggsae orthologs were also differentially expressed. Of those, 29 were shared between the three conditions: Orsay virus [N2], Orsay virus [rde-1], and Santeuil virus [JU1264]. Strikingly, 14 of the 29 genes were members of a single gene family, the $\mathrm{C} 17 \mathrm{H} 1$ family genes in C. elegans. Induction of members of this gene family in response to viral infection was conserved in two divergent Caenorhabditis nematode species despite $\sim 18$ million years of host evolution. Furthermore, analysis of other published transcriptomes identified induction of $\mathrm{C} 17 \mathrm{H} 1$ family genes by bacterial and fungal pathogens. The upregulation of a subset of these genes by disparate microbes such as virus, bacteria, and fungi raises the possibility that this gene family may form the core of a pan-microbial stress response. To date, there has been no reported function associated with these family members. The large number of paralogs induced following Orsay virus or $N$. parisii infection suggests the possibility of functional redundancy, which would provide a challenge in experimental testing of the functions of these genes.

\section{Conclusions}

Our transcriptional profiling study of both virus and microsporidium infection provides insights into the host response to pathogens. We found that distinct pathogens such as Orsay virus and $N$. parisii elicited a similar set of DEGs in C. elegans, suggesting that these DEGs may constitute a broad pan-microbial response to infection. Additionally, within the transcriptional profile of viral infection in the two different nematode species $C$. elegans and C. briggsae, we found a shared set of 58 evolutionarily conserved transcriptional responsive genes to viral infection, many of which have no known function. Given the fact that diverse hosts regulate these common genes in response to distinct viral infections suggests that they play important roles. Further studies are needed to define the impact and mechanism of action of these genes on viral infection.

\section{Methods \\ Strains}

N2 and rde-1 (WM27) were obtained from the Caenorhabditis Genetics Center (CGC). Isolation of wild C. briggsae strains JU1264 has been described [18].

\section{Infectious virus filtrate preparation}

Orsay virus and Santeuil virus were propagated as previously described [18]. Briefly, C. elegans rde-1 mutants were subjected to Orsay virus infection. C. briggsae JU1264 were subjected to Santeuil virus infection. Infected animals were subsequently collected and homogenized. The homogenate were passed through a $0.22 \mu \mathrm{m}$ filter to obtain filtered viruses.

\section{Quantification of virus titer}

To measure the infectious titer of viruses, we employed a method similar to tissue culture infectious dose $50 \%$ $\left(\mathrm{TCID}_{50}\right)$ using live C. elegans or C. briggsae in wells instead of cultured cells. We were not able to measure killing of nematodes as none of the viruses were lethal. To measure infectivity in a well, we used qRT-PCR to determine whether replication of viral RNA occurred, using a criterion of $\mathrm{Ct}$ value of 30 and below as positive infection. Animals were synchronized and plated on 6well plate seeded with $20 \mu \mathrm{l}$ OP50 food. Virus filtrates were serially diluted 10 -fold to $10^{-8} .20 \mu \mathrm{l}$ of each dilution were added to a well containing animals and combined to have four total replicates per condition. 
Infected animals were incubated at $20{ }^{\circ} \mathrm{C}$ for three days and collected into Trizol. RNA samples were extracted using Zymo 96-well RNA extraction kit. The stock Orsay virus filtrate had a titer of $2.3 \times 10^{6} \mathrm{TCID}_{50} / \mathrm{ml}$ and Santeuil virus had a titer of $8.9 \times 10^{6} \mathrm{TCID}_{50} / \mathrm{ml}$.

\section{Pathogen inoculation}

Three independent infections were conducted for each strain and pathogen. Two methods were used to infect animals. 1) Uninfected C. elegans (N2 and $r d e-1)$ and $C$. briggsae (wild isolate JU1264) were synchronized by standard bleach treatment. 2,000 embryos were seeded per well into 6-well NGM plates containing $20 \mu \mathrm{l}$ of OP50 food and maintained at $20{ }^{\circ} \mathrm{C}$. For each condition, 18 wells were prepared and collected. $39 \mathrm{~h}$ after bleaching, animals (L3 stage) were inoculated with $20 \mu \mathrm{l}$ of Orsay virus $\left(4.0 \times 10^{5} \mathrm{TCID}_{50} / \mathrm{ml}\right)$ for C. elegans or Santeuil virus $\left(8.9 \times 10^{5} \mathrm{TCID}_{50} / \mathrm{ml}\right)$ for $C$. briggsae, or M9 buffer as control. $12 \mathrm{~h}$ post infection, animals were rinsed off from the wells with $1 \mathrm{ml} \mathrm{M9}$ buffer, supernatant were removed after centrifugation and $1 \mathrm{ml}$ Trizol were added. 2) Uninfected C. elegans (N2 and rde-1) were synchronized by standard bleach treatment and 20,000 embryos were added to $10 \mathrm{~cm}$ NGM plates seeded with $1.5 \mathrm{ml}$ of OP50. $39 \mathrm{~h}$ after bleaching, animals (L3 stage) were inoculated with either $200 \mu$ l Orsay virus $\left(4.0 \times 10^{5} \mathrm{TCID}_{50} / \mathrm{ml}\right), N$. parisii microsporidia $(10,000$ spores/animal), or homogenates of uninfected $r d e-1$ passed through a $0.22 \mu \mathrm{m}$ pore filter (mock control). C. briggsae (JU1264) were treated similarly and infected with $200 \mu$ l Santeuil $\left(8.9 \times 10^{5} \mathrm{TCID}_{50} / \mathrm{ml}\right)$ virus. Animals were rinsed off from the plates and harvested in Trizol at $12 \mathrm{~h}$ after exposure to virus, microsporidia, or mock control.

\section{Preparation of RNA-seq sample}

Total RNA was extracted using Trizol and mRNA was subsequently enriched using OligoTex mRNA mini (Qiagen) according to the manufacturer's protocol. RNA concentrations were assessed by Qubit fluorimeter (Life Technologies) and 10-100 ng of each sample were sent to Genome Technology Access Center in the Department of Genetics at Washington University School of Medicine (GTAC) for RNA-Seq.

\section{RNA-Seq Analysis}

Illumina HiSeq platform-generated single end reads of 50 bp were aligned to N2 reference strain and AF16 reference strain transcriptomes (WS250) with TopHat2 [40]. The aligned reads were counted with HT-Seq [41]. Only uniquely aligned reads were counted and used for downstream analysis. The read counts were processed with $\mathrm{R}$ package edgeR $[28,42]$. Samples were subjected to batch effect adjustment as there were two different methods of preparation and all three replicates were independently conducted on different days. The resulting counts were subjected to standard edgeR differential expressed gene analysis with a statistical cutoff of FDR $<0.05$.

\section{Real time quantitative two-step RT-PCR (qRT-PCR) of host genes}

$1 \mu \mathrm{g}$ of total RNA from each sample was treated with DNaseI (Fermentus) according to the manufacture's protocol, purified using an RNeasy kit (Invitrogen) and then eluted in $20 \mu \mathrm{l}$ RNase/DNase free water. cDNA synthesis was performed by using an oligo(dT) primer with thermoscript reverse transcriptase (Thermo Scientific) at $65{ }^{\circ} \mathrm{C}$ for $45 \mathrm{~min}$. The synthesized cDNA was diluted 1:10 and $5 \mathrm{ul}$ of the diluted cDNA was used for real time-qPCR. Real time qPCR was performed using Taqman qPCR master mix reagents (Applied Biosystem) on a ViiA7 real time PCR system (Applied Biosystem) following the manufacturer's suggested protocol. Each analyzed gene was normalized to an internal control $c d c-42$ gene and expressed as fold change of infected samples compared to mocked infected samples.

\section{Association of bacterial or fungal pathogen DEGs with Orsay virus DEGs}

The significance of association between bacterial or fungal DEGs from previous studies and Orsay virus DEGs was measured by a Fisher exact test. Briefly, concordant (upregulated in both or down-regulated in both) DEGs between a selected pathogen and Orsay virus were counted as the overlapped DEGs. Fisher exact test was calculated with the total non-overlapped pathogen DEGs, the non-overlapped Orsay virus DEGs and all other remaining genes (non-differentially expressed following bacterial, fungal or viral infection). Pair-wise comparison between pathogens DEGs against Orsay RNA were done for all pathogens.

\section{Additional files}

\footnotetext{
Additional file 1: Heatmap of differentially expressed genes upon pathogen infections. The heatmap showed the expression level for all of the differentially expressed genes in the three infection conditions (Orsay virus [N2], Orsay virus [rde-1], and N. parisii [N2]). A) $\log _{2}$ CPM of each gene presented. B) Median normalized $\log _{2}$ CPM of each gene. Each CPM value was normalized to the median CPM for the given gene. Each experimental condition had three replicates and each replicate was represented in a column. Samples that did not have measurable expression were grey. (PDF $1297 \mathrm{~kb}$ )

Additional file 2: List of differentially expressed genes upon pathogen infections identified by edgeR. (XLSX $75 \mathrm{~kb}$ )

Additional file 3: Confirmation of C. elegans RNA-seq with qRT-PCR. Expression of $\mathrm{N} 2$ response genes to Orsay virus infection with RNA-seq was confirmed with qRT-PCR. qRT-PCR results were normalized to $c d c-42$ before calculating fold-change. (PDF $29 \mathrm{~kb}$ )
} 
Additional file 4: Comparison of DEGs with previous publications. Venn diagrams showed the comparison of DEGs from our current study to previous published studies. A) Comparison of up-regulated DEGs from $\mathrm{N}$. parisii [N2] infection. The most similar conditions from Bakowski et al. were used ( $8 \mathrm{~h}$ and $16 \mathrm{~h}$ post $\mathrm{N}$. parisii infection). B) Comparison of up-regulated genes in N2 after Orsay virus infection between current study and Sarkies et al. C) Comparison between down-regulated genes in N2 after Orsay virus infection between current study and Sarkies et al. D) Comparison between up-regulated genes in rde-1 after Orsay virus infection between current study and Sarkies et al. E) Comparison between down-regulated genes in rde-1 after Orsay virus infection between current study and Sarkies et al. (PDF $462 \mathrm{~kb}$ )

Additional file 5: List of differentially expressed genes compared to previous publications. (XLSX $24 \mathrm{~kb}$ )

Additional file 6: Confirmation of C. briggsae RNA-seq with qRT-PCR. Expression level from RNA-seq of JU1264 response genes to Santeuil virus infection was confirmed with qRT-PCR. qRT-PCR results were normalized to $c b r-c d c-42$ before calculating fold-change. (PDF $28 \mathrm{~kb}$ )

Additional file 7: Summary of Orsay virus DEGs in C. elegans that have C. briggsae orthologs DEGs by Santeuil virus infection. (XLSX $20 \mathrm{~kb}$ )

\section{Abbreviations}

ALS2CR12: Amyotrophic Lateral Sclerosis 2 Chromosome Region Candidate 12; CPM: Counts per million; DAVID: Database for Annotation, Visualization, and Integrated Discovery; DEGs: Differentially expressed genes; MATH: Meprin-associated Traf homology; qRT-PCR: Quantitative real-time reverse transcription PCR; RNAi: RNA interference; RNA-seq: RNA sequencing; SCF complex: Skp/Cullin/F-box complex

\section{Acknowledgements}

We thank Emily Troemel for providing N. parisii. This research was supported by NIH R21 Al097865. D.W. holds an Investigator in the Pathogenesis of Infectious Disease award from the Burroughs Wellcome Fund. The N2 and WM27 strains were provided by the Caenorhabditis genetic center (CGC), which is funded by NIH Office of Research Infrastructure Programs (P40 OD010440). We thank the Genome Technology Access Center in the Department of Genetics at Washington University School of Medicine for help with genomic analysis. The Center is partially supported by NCl Cance Center Support Grant \#P30 CA91842 to the Siteman Cancer Center and by ICTS/CTSA Grant\# UL1 TR000448 from the National Center for Research Resources (NCRR), a component of the National Institutes of Health (NIH), and NIH Roadmap for Medical Research. This publication is solely the responsibility of the authors and does not necessarily represent the official view of $\mathrm{NCRR}$ or $\mathrm{NIH}$.

\section{Funding}

This research was supported by NIH R21 Al097865. D.W. holds an Investigator in the Pathogenesis of Infectious Disease award from the Burroughs Wellcome Fund.

\section{Availability of data and materials}

The datasets supporting the conclusions of this article are available in the NCBI BioProject repository (BioProject Accession number: PRJNA352987; http://www.ncbi.nlm.nih.gov/bioproject/352987) and in the SRA database (SRA Accession number: SRP100798; https://www.ncbi.nlm.nih.gov//sra/ ?term $=$ SRP100798).

\section{Authors' contributions}

$\mathrm{KC}, \mathrm{CF}, \mathrm{HJ}$ and $\mathrm{YJ}$ performed the infection and RNA-seq. $\mathrm{KC}$ and $\mathrm{HJ}$ performed the molecular biology experiments. $\mathrm{KC}$ performed the computational analysis. KC, CF and DW wrote the manuscript. All authors read and approved the final manuscript.

\section{Competing interests}

The authors declare that they have no competing interests.

\section{Consent for publication}

Not applicable.

\section{Ethics approval and consent to participate}

Not applicable (Animal ethics approval is not required for studies with invertebrate nematodes. Nematode strains were obtained through the Caenorhabditis genetic center).

\section{Publisher's Note}

Springer Nature remains neutral with regard to jurisdictional claims in published maps and institutional affiliations.

Received: 1 December 2016 Accepted: 7 April 2017 Published online: 17 April 2017

\section{References}

1. Irazoqui JE, Urbach JM, Ausubel FM. Evolution of host innate defence: insights from Caenorhabditis elegans and primitive invertebrates. Nat Rev Immunol. 2010;10:47-58. Nature Publishing Group.

2. Marsh EK, May RC. Caenorhabditis elegans, a model organism for investigating immunity. Appl Environ Microbiol. 2012;78:2075-81.

3. Kim DH, Feinbaum R, Alloing G, Emerson FE, Garsin DA, Inoue H, et al. A conserved p38 MAP kinase pathway in Caenorhabditis elegans innate immunity. Science. 2002;297:623-6

4. Mallo GV, Le C, Couillault C, Pujol N, Granjeaud S, Kohara Y, et al. Inducible Antibacterial Defense System in C. elegans. Curr Biol. 2002;12:1209-14.

5. Garsin D, Villanueva J, Begun J, Kim D, Sifri C, Calderwood S, et al. LongLived C. elegans daf-2 Mutants Are Resistant to Bacterial Pathogens. Science. 2003:300:1921.

6. Estes KA, Dunbar TL, Powell JR, Ausubel FM, Troemel ER. bZIP transcription factor zip-2 mediates an early response to Pseudomonas aeruginosa infection in Caenorhabditis elegans. Proc Natl Acad Sci U S A. 2010;107:2153-8.

7. Boehnisch C, Wong D, Habig M, Isermann K, Michiels NK, Roeder T, et al. Protist-type lysozymes of the nematode Caenorhabditis elegans contribute to resistance against pathogenic Bacillus thuringiensis. PLoS One. 2011;6.

8. Irazoqui JE, Troemel ER, Feinbaum RL, Luhachack LG, Cezairliyan BO, Ausubel FM. Distinct pathogenesis and host responses during infection of C. elegans by P. aeruginosa and S. aureus. PLoS Pathog. 2010;6:e1000982.

9. Wong D, Bazopoulou D, Pujol N, Tavernarakis N, Ewbank JJ. Genome-wide investigation reveals pathogen-specific and shared signatures in the response of Caenorhabditis elegans to infection. Genome Biol. 2007;8:R194.

10. Pujol N, Zugasti O, Wong D, Couillault C, Kurz CL, Schulenburg H, et al. Antifungal innate immunity in C. elegans is enhanced by evolutionary diversification of antimicrobial peptides. PLoS Pathog. 2008;4:e1000105.

11. Engelmann I, Griffon A, Tichit L, Montañana-Sanchis F, Wang G, Reinke V, et al. A comprehensive analysis of gene expression changes provoked by bacterial and fungal infection in C. elegans. PLoS One. 2011;6:e19055.

12. Bakowski MA, Desjardins CA, Smelkinson MG, Dunbar TA, Lopez-Moyado IF, Rifkin SA, et al. Ubiquitin-mediated response to microsporidia and virus infection in C. elegans. Schneider DS, editor. PLoS Pathog. Public Library of Science; 2014;10:e1004200.

13. Yang $W$, Dierking $K$, Rosenstiel PC, Schulenburg H. GATA transcription factor as a likely key regulator of the Caenorhabditis elegans innate immune response against gut pathogens. Zoology. 2016;119(4):244-53.

14. Liu W-H, Lin Y-L, Wang J-P, Liou W, Hou RF, Wu Y-C, et al. Restriction of vaccinia virus replication by a ced-3 and ced-4-dependent pathway in Caenorhabditis elegans. Proc Natl Acad Sci U S A. 2006;103:4174-9.

15. Wilkins C, Dishongh R, Moore SC, Whitt MA, Chow M, Machaca K. RNA interference is an antiviral defence mechanism in Caenorhabditis elegans. Nature. 2005:436:1044-7.

16. Schott DH, Cureton DK, Whelan SP, Hunter CP. An antiviral role for the RNA interference machinery in Caenorhabditis elegans. Proc Natl Acad Sci U S A. 2005;102:18420-4

17. Lu R, Maduro M, Li F, Li HW, Broitman-Maduro G, Li WX, et al. Animal virus replication and RNAi-mediated antiviral silencing in Caenorhabditis elegans. Nature. 2005;436:1040-3

18. Félix M-A, Ashe A, Piffaretti J, Wu G, Nuez I, Bélicard T, et al. Natural and experimental infection of Caenorhabditis nematodes by novel viruses related to nodaviruses. PLoS Biol. 2011;9, e1000586.

19. Sarkies P, Ashe A, Le Pen J, McKie MA, Miska EA. Competition between virus-derived and endogenous small RNAs regulates gene expression in Caenorhabditis elegans. Genome Res. 2013;23:1258-70. 
20. Ashe A, Bélicard T, Le Pen J, Sarkies P, Frézal L, Lehrbach NJ, et al. A deletion polymorphism in the Caenorhabditis elegans RIG-I homolog disables viral RNA dicing and antiviral immunity. Elife. 2013;2013:1-21.

21. Guo X, Zhang R, Wang J, Ding S-W, Lu R. Homologous RIG--like helicase proteins direct RNAi-mediated antiviral immunity in C. elegans by distinct mechanisms. Proc Natl Acad Sci U S A. 2013;110:16085-90.

22. Sterken MG, Snoek LB, Bosman KJ, Daamen J, Riksen JAG, Bakker J, et al. A heritable antiviral RNAi response limits Orsay virus infection in Caenorhabditis elegans N2. Aballay A, editor. PLoS One. Public Library of Science; 2014;9:e89760

23. Ashe A, Sarkies P, Le Pen J, Tanguy M, Miska EA. Antiviral RNAi against Orsay virus is neither systemic nor transgenerational in Caenorhabditis elegans. J Virol. 2015:doi:10.1128/JVI.03664-14.

24. Franz CJ, Zhao G, Félix M-A, Wang D. Complete genome sequence of Le Blanc virus, a third Caenorhabditis nematode-infecting virus. J Virol. 2012;86:11940.

25. Franz CJ, Renshaw H, Frezal L, Jiang Y, Félix M-A, Wang D. Orsay, Santeuil and Le Blanc viruses primarily infect intestinal cells in Caenorhabditis nematodes. Virology. 2014;448:255-64.

26. Cutter AD. Divergence Times in Caenorhabditis and Drosophila Inferred from Direct Estimates of the Neutral Mutation Rate. Mol Biol Evol. 2008;25:778-86. Oxford University Press.

27. Whiteman NK, Barrie A, Ausubel FM. Microsporidia Are Natural Intracellular Parasites of the Nematode Caenorhabditis elegans. 2008;6.

28. McCarthy DJ, Chen Y, Smyth GK. Differential expression analysis of multifactor RNA-Seq experiments with respect to biological variation. Nucleic Acids Res. 2012;40:4288-97.

29. Huang DW, Lempicki RA, Sherman BT. Systematic and integrative analysis of large gene lists using DAVID bioinformatics resources. Nat Protoc. 2009;4: 44-57. Nature Publishing Group.

30. Huang DW, Sherman BT, Lempicki RA. Bioinformatics enrichment tools: paths toward the comprehensive functional analysis of large gene lists. Nucleic Acids Res. 2009;37:1-13. Oxford University Press.

31. Dong M-Q, Venable JD, Au N, Xu T, Park SK, Cociorva D, et al. Quantitative mass spectrometry identifies insulin signaling targets in C. elegans. Science. 2007:317:660-3. American Association for the Advancement of Science.

32. Ding YH, Du YG, Luo S, Li YX, Li TM, Yoshina S, et al. Characterization of PUD-1 and PUD-2, Two Proteins Up-Regulated in a Long-Lived daf-2 Mutant. Ma J, editor. PLoS One. Public Library of Science; 2013;8:e67158.

33. Thomas $\mathrm{JH}$. Adaptive evolution in two large families of ubiquitin-ligase adapters in nematodes and plants. Genome Res. 2006;16:1017-30. Cold Spring Harbor Laboratory Press.

34. Stein LD, Bao Z, Blasiar D, Blumenthal T, Brent MR, Chen N, et al. The Genome Sequence of Caenorhabditis briggsae: A Platform for Comparative Genomics. Jonathan A. Eisen, editor. PLoS Biol. Public Library of Science; 2003;1:e45.

35. Laguette N, Sobhian B, Casartelli N, Ringeard M, Chable-Bessia C, Ségéral E, et al. SAMHD1 is the dendritic- and myeloid-cell-specific HIV-1 restriction factor counteracted by Vpx. Nature. 2011;474:654-7. Europe PMC Funders.

36. Evans EA, Kawli T, Tan M-W. Pseudomonas aeruginosa Suppresses Host Immunity by Activating the DAF-2 Insulin-Like Signaling Pathway in Caenorhabditis elegans. Ausubel FM, editor. PLoS Pathog. Public Library of Science; 2008;4:e1000175.

37. Cuomo CA, Desjardins CA, Bakowski MA, Goldberg J, Ma AT, Becnel JJ, et al. Microsporidian genome analysis reveals evolutionary strategies for obligate intracellular growth. Genome Res. 2012;22:2478-88. Cold Spring Harbor Laboratory Press.

38. Sato K, Yoshiga T, Hasegawa K. Activated and inactivated immune responses in Caenorhabditis elegans against Photorhabdus luminescens TT01. Springerplus. 2014;3:274. Springer.

39. Jansson HB. Adhesion of Conidia of Drechmeria coniospora to Caenorhabditis elegans Wild Type and Mutants. J Nematol. 1994;26:430-5.

40. Kim D, Pertea G, Trapnell C, Pimentel H, Kelley R, Salzberg SL, et al. TopHat2: accurate alignment of transcriptomes in the presence of insertions, deletions and gene fusions. Genome Biol. 2013;14:R36. BioMed Central.

41. Anders S, Pyl PT, Huber W. HTSeq-a Python framework to work with high-throughput sequencing data. Bioinformatics. 2015;31:166-9. Oxford University Press.

42. Robinson MD, McCarthy DJ, Smyth GK. edgeR: A Bioconductor package for differential expression analysis of digital gene expression data. Bioinformatics. 2009;26:139-40.

\section{Submit your next manuscript to BioMed Central and we will help you at every step:}

- We accept pre-submission inquiries

- Our selector tool helps you to find the most relevant journal

- We provide round the clock customer support

- Convenient online submission

- Thorough peer review

- Inclusion in PubMed and all major indexing services

- Maximum visibility for your research

Submit your manuscript at www.biomedcentral.com/submit
Biomed Central 